\title{
CARMENES: data flow
}

J. A. Caballero*a,b , J. Guàrdiac ${ }^{\mathrm{c}}$, M. López del Fresno ${ }^{\mathrm{b}}$, M. Zechmeister ${ }^{\mathrm{d}}$, E. de Juan ${ }^{\mathrm{e}}$, F. J. Alonso-Floriano ${ }^{f}$, P. J. Amado ${ }^{g}$, J. Colomé ${ }^{c}$, M. Cortés-Contreras ${ }^{f}$, Á. García-Piquer $^{c}$, L. Gesa $^{c}$, E. de Guindos ${ }^{\mathrm{e}}$, H.-J. Hagen ${ }^{\mathrm{h}}$, J. Helmling ${ }^{\mathrm{e}}$, L. Hernández Castaño ${ }^{\mathrm{e}}$, M. Kürster ${ }^{\mathrm{i}}, \mathrm{J}_{\text {. López-Santiago }}{ }^{f}$, D. Montes ${ }^{\mathrm{f}}$, R. Morales Muñoz ${ }^{\mathrm{g}}$, A. Pavlovi, A. Quirrenbach ${ }^{\mathrm{a}}$, A. Reiners ${ }^{\mathrm{d}}$, I. Ribas ${ }^{\mathrm{c}}$, W. Seifert ${ }^{\mathrm{a}}$, E. Solano ${ }^{\mathrm{b}}$

${ }^{\mathrm{a}}$ Landessternwarte (ZAH), Königstuhl 12, D-69117 Heidelberg, Germany; ${ }^{\mathrm{b}}$ Centro de Astrobiología (CSIC-INTA), Campus ESAC, Camino Bajo del Castillo s/n, E-28692 Villanueva de la Cañada, Madrid, Spain; 'Institut de Ciències de l'Espai (CSIC-IEEC), Campus UAB, c/ de Can Magrans s/n,

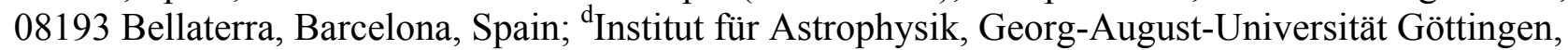

Friedrich-Hund-Platz 1, D-37077 Göttingen, Germany; ${ }^{\mathrm{e} C e n t r o}$ Astronómico Hispano-Alemán (MPG-CSIC), Observatorio Astronómico de Calar Alto, Sierra de los Filabres, E-04550 Gérgal, Almería, Spain; ${ }^{f}$ Departamento de Astrofísica, Facultad de Física, Universidad Complutense de Madrid, E-28040 Madrid, Spain; ${ }^{\mathrm{g}}$ Instituto de Astrofísica de Andalucía (CSIC), Glorieta de la Astronomía s/n, E-18008 Granada, Spain; ${ }^{h}$ Hamburger Sternwarte, Gojenbergsweg 112, D-21029 Hamburg, Germany; ${ }^{\mathrm{i} M a x-P l a n c k-I n s t i t u t ~ f u ̈ r ~ A s t r o n o m i e, ~ K o ̈ n i g s t u h l ~ 17, ~ D-69117 ~ H e i d e l b e r g, ~}$ Germany

\begin{abstract}
CARMENES, the new Calar Alto spectrograph especially built for radial-velocity surveys of exoearths around $\mathrm{M}$ dwarfs, is a very complicated system. For reaching the goal of $1 \mathrm{~m} / \mathrm{s}$ radial-velocity accuracy, it is appropriate not only to monitor stars with the best observing procedure, but to monitor also the parameters of the CARMENES subsystems and safely store all the engineer and science data. Here we describe the CARMENES data flow from the different subsystems, through the instrument control system and pipeline, to the virtual-observatory data server and astronomers.
\end{abstract}

Keywords: Site operations, data networks, computers, instrument control systems, pipelines, astronomical databases

\section{INTRODUCTION}

In general, scientists show their greatest interest in imposing the top-level requirements in the conceptual and preliminary design phases at the beginning of an instrumental project, and in retrieving and analysing the output data during the science exploitation phase at the end of the project. Meanwhile, optical, mechanical, electric and software engineers focus their attention in some of the intermediate steps of the project, from the preliminary and final design phases, through the manufacture, assembly, integration and verification phase, to the instrument commissioning. As a result, only a few individuals (e.g., Principal Investigator, Project Scientist, System Engineer, Project Manager) participate in all steps of the instrumental project. In the case of a complex instrument in which the science results are sensitive to tiny variations in any of the different subsystems, an end-to-end supervision of the data flow is indispensable.

CARMENES stands for Calar Alto high-Resolution search for M dwarfs with Exoearths with Near-infrared and optical Echelle Spectrographs. It is an ultra-stabilised double-channel spectrograph that covers in one shot from $0.52 \mu \mathrm{m}$ to 1.71 $\mu \mathrm{m}$ with a spectral resolution greater than 80,000 (Quirrenbach et al., this volume). Its front-end is installed in the Cassegrain focus of the $3.5 \mathrm{~m}$ Zeiss telescope of the Calar Alto observatory (Centro Astronómico Hispano-Alemán, CAHA) in Almería, in the south of Spain. Its two fibre-fed spectrographs, dubbed VIS and NIR, are inside climate

*ja.caballero@csic.es; http://carmenes.caha.es

Observatory Operations: Strategies, Processes, and Systems VI, edited by Alison B. Peck, Robert L. Seaman, Chris R. Benn, Proc. of SPIE Vol. 9910, 99100E · @ 2016 SPIE · CCC code: 0277-786X/16/\$18 · doi: 10.1117/12.2233574 
chambers in the $3.5 \mathrm{~m}$ telescope coudé room, together with the vacuum, cooling and wavelength calibration systems, and the main computers. To date, CARMENES is the only precise high-resolution double spectrograph with a wide wavelength coverage in both optical and near infrared that delivers $\sim 1 \mathrm{~m} \mathrm{~s}^{-1}$ radial-velocity precision (some on-going projects with the same goal are GIARPS = GIANO+HARPS-N at the $3.6 \mathrm{~m}$ Telescopio Nazionale Galileo in La Palma, NIRPS+HARPS-S at the $3.6 \mathrm{~m}$ ESO telescope in La Silla and, at a longer timescale, HiRes at the $39 \mathrm{~m}$ European Extremely Large Telescope).

CARMENES was designed and built by the eponymous CARMENES consortium, which consists of ten astronomical research centres and universities in Spain and Germany and the Spanish-German Calar Alto observatory. This relatively large number of consortium members with different expertises (e.g., near-infrared detectors, vacuum and cryogenics, interlocks, instrument control system, scheduling, data pipelining, archiving) translated into numerous interfaces between subsystems, which required a close and constant monitorisation by a small team of astronomers and engineers during the design, MAIV and commissioning phases.

The instrument complexity is illustrated by the list of 15 CARMENES computers (including main and spare) in Table 1. The CARMENES computers are named after astronomers that (have) contributed significantly to the discovery of $\mathrm{M}$ dwarfs ${ }^{1}$. All of them run on Linux openSUSE 12.364 bits, except the NIR computer, which runs on Linux openSUSE 13.164 bits.

Table 1. List of CARMENES computers at the Calar Alto observatory.

\begin{tabular}{|c|c|c|c|}
\hline Computer & Task & Model & Location \\
\hline $\begin{array}{l}\text { ICS Lab } \\
\text { (luyten) }\end{array}$ & $\begin{array}{l}\text { ICS GUI, web (CAHA } \\
\text { weather, finding charts, } \\
\text { Carmencita, Simbad, ADS, } \\
\text { skype) }\end{array}$ & $\begin{array}{l}\text { Dell OptiPlex } 7010 \text { MT desktop, i7 Quad Core, } \\
\text { 1TB storage }\end{array}$ & $\begin{array}{l}\text { Remote } \\
\text { observing } \\
\text { room }\end{array}$ \\
\hline $\begin{array}{l}\text { ICS One } \\
\text { (gliese) }\end{array}$ & \multirow{2}{*}{$\begin{array}{l}\text { Instrument control and } \\
\text { monitoring, scheduling, } \\
\text { nightly spectra storage }\end{array}$} & \multirow{2}{*}{$\begin{array}{l}\text { Dell PowerEdge R420 rack server, } 2 \text { x Intel Xeon } \\
\text { E5-2430 v2 ( } 6 \text { cores, } 2.5 \mathrm{GHz}), 2 \mathrm{~TB} \text { internal } \\
\text { storage }+2 \mathrm{~TB} \text { external storage, 16GB RAM, 1U } \\
\text { rack chassis }\end{array}$} & \multirow[t]{2}{*}{$\begin{array}{l}\text { Coudé room } \\
\text { main rack }\end{array}$} \\
\hline $\begin{array}{l}\text { ICS Two } \\
\text { (jahreiss) }\end{array}$ & & & \\
\hline $\begin{array}{l}\text { NIR pipe Lab } \\
\text { (lacaille) }\end{array}$ & \multirow[t]{2}{*}{ Pipeline GUIs, night logs, wiki } & \multirow[t]{2}{*}{$\begin{array}{l}\text { Dell OptiPlex } 9020 \text { MT desktop, i5 Quad Core, } \\
\text { 500GB storage, 8GB RAM }\end{array}$} & \multirow{2}{*}{$\begin{array}{l}\text { Remote } \\
\text { observing } \\
\text { room }\end{array}$} \\
\hline $\begin{array}{l}\text { VIS pipe Lab } \\
\text { (kapteyn) }\end{array}$ & & & \\
\hline $\begin{array}{l}\text { NIR pipeline } \\
\text { (lalande) }\end{array}$ & \multirow[t]{2}{*}{ First pipeline (CARACAL) } & \multirow{2}{*}{$\begin{array}{l}\text { Dell PowerEdge R420 rack server, } 2 \text { Intel Xeon } \\
\text { E5-2430 v2 ( } 6 \text { cores, } 2.5 \mathrm{GHz}) \text {, 2TB storage, } \\
\text { 16GB RAM, } 1 \mathrm{U} \text { rack chassis }\end{array}$} & \multirow[t]{2}{*}{$\begin{array}{l}\text { Coudé room } \\
\text { main rack }\end{array}$} \\
\hline $\begin{array}{l}\text { VIS pipeline } \\
\text { (barnard) }\end{array}$ & & & \\
\hline $\begin{array}{l}\text { NIR comp } \\
(\text { ross } x 2)\end{array}$ & $\begin{array}{l}\text { GEIRS/NIR detector and } \\
\text { readout, NIR exp-meter, NIR } \\
\text { fibre shaker }\end{array}$ & Dell PowerEdge R720 rack server & NIR rack \\
\hline $\begin{array}{l}\text { VIS comp } \\
\text { (wolf } x 2 \text { ) }\end{array}$ & $\begin{array}{l}\text { VIS detector, VIS exp-meter, } \\
\text { VIS fibre shaker, VIS shutter } \\
\text { in front-end }\end{array}$ & Dell PowerEdge R420 rack server & VIS rack \\
\hline $\begin{array}{l}\text { A\&G comp } \\
\text { (giclas x2) }\end{array}$ & A\&G camera control & cirrus $^{7}$ nimbus & Front-end \\
\hline $\begin{array}{l}\text { Interlocks } \\
\text { (struve x2) }\end{array}$ & $\begin{array}{l}\text { Alarms display, Scada/Mango } \\
\text { access and control of pumps }\end{array}$ & $\begin{array}{l}\text { Dell OptiPlex } 7010 \text { desktop, i5 Quad Core, } \\
\text { 3.2GHz, } 8 \text { GB RAM, 1TB storage }\end{array}$ & $\begin{array}{l}\text { Coudé room } \\
\text { main rack }\end{array}$ \\
\hline
\end{tabular}

\footnotetext{
${ }^{1}$ N.-L. de Lacaille (1713), J. J. L. de Lalande (1732), F. G. V. von Struve (1793), J. C. Kapteyn (1851), E. E. Barnard (1857), M. Wolf (1863), F. E. Ross (1874), W. J. Luyten (1899), H. L. Giclas (1910), W. Gliese (1915), and H. Jahreiss (1942).
} 
This contribution deals with the CARMENES data flow, where "data" here stands for any collection of alpha-numeric or Boolean values that are needed for the final scientific result, which for our guaranteed time observations (GTO) is the discovery and characterisation of exoplanets around nearby, cool, dwarf stars of M spectral type. Some of these planets may have masses and radii similar to the Earth's, and may be as well inside the habitable zones around their host stars. This contribution is complementary to other ones presented by CARMENES members in SPIE Astronomical Telescopes and Instrumentation 2016, such as the general instrument overview by Quirrenbach et al. (9908-38) and descriptions of the VIS channel by Seifert et al. (9908-231), the NIR channel by Becerril et al. (9910-32), and project management and system engineering by García-Vargas et al. (9911-24) and Pérez-Calpena (9911-78). This data flow description is inseparable from the CARMENES contributions on interlocks by Helmling et al. (9908-237) and, especially, instrument control software by Colomé et al. (9913-149).

\section{DATA FLOW}

\subsection{Input catalogue (Carmencita)}

Carmencita, the CARMEN[ES] Cool star Information and daTa Archive, is the M-dwarf database from where we chose our best target sample (Caballero et al. 2013; Quirrenbach et al. 2015). As part of our GTO project, about 300 late-type M dwarfs are monitored by CARMENES from Calar Alto during at least 600 nights in the 2016-2018 timeframe (García-Piquer et al. 2016). Carmencita catalogues over 2000 carefully-selected $M$ dwarfs northern of $\delta=-23$ deg. For each star, we tabulate dozens of parameters (accurate astrometry, spectral typing, photometry in 20 bands from the ultraviolet to the mid-infrared, rotational and radial velocities, X-ray count rates and hardness ratios, close and wide multiplicity data and many more) compiled from the literature or measured by us with new data. Carmencita is perhaps the most comprehensive database of bright, nearby, M dwarfs (Fig. 1).
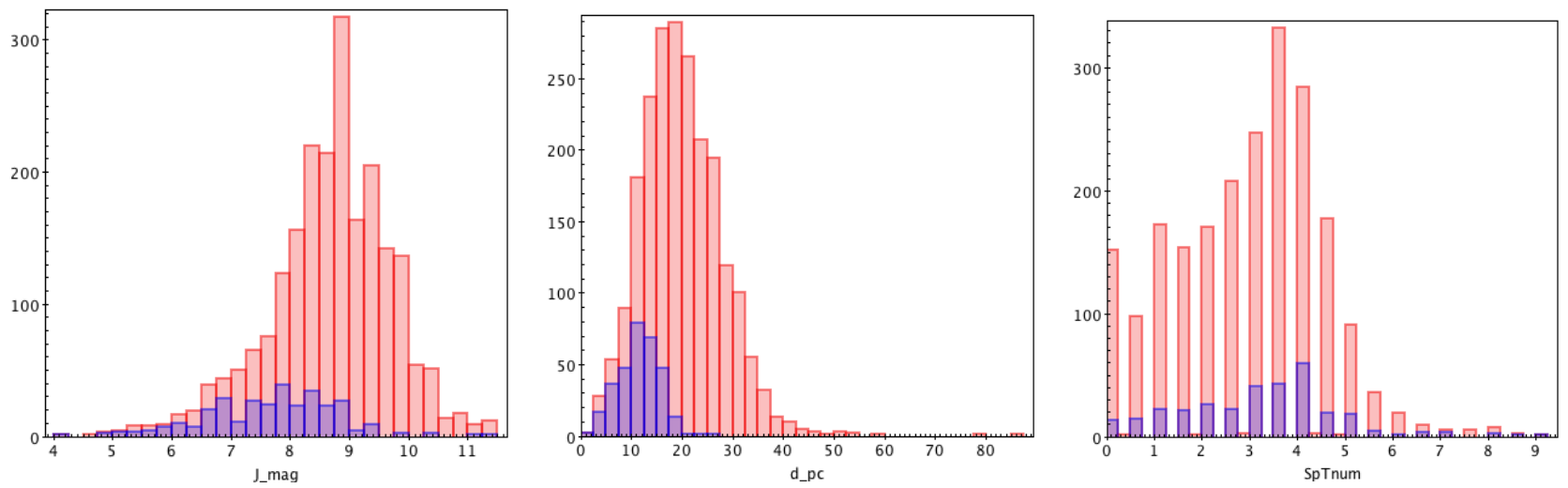

Figure 1: Distribution of $J$ magnitudes (left), heliocentric distances (centre) and spectral types (right) of the $\sim 2200$ Carmencita stars (red) and the $\sim 300$ GTO targets (blue). Mean magnitudes, distances and spectral types of the GTO sample are $7.7 \mathrm{mag}, 11.6 \mathrm{pc}$ and $\mathrm{M} 3.0 \mathrm{~V}$, respectively.

As illustrated by Fig. 2, Carmencita is fed by a number of literature sources (Joy \& Abt 1974; Lee 1984; Bidelmean 1985; Henry et al. 1994; Reid et al. 1995; Scholz et al. 2005; Lépine \& Gaidos 2011; Lépine et al. 2013, etc.), public allsky surveys (Perryman et al. 1997; Mason et al. 2001; Skrutskie et al. 2006; Roeser et al. 2010) and our own preparatory observations with FastCam/1.5 m TCS (high-resolution imaging), CAFOS/2.2 m CAHA (low-resolution spectroscopy), FEROS $2.2 \mathrm{~m}$ La Silla, CAFÉ/2.2 m CAHA, HRS/9.4 m HET (high-resolution spectroscopy).

All the stars and their parameters are methodically ingested into an ascii file saved with the CSV extension for internal distribution, and visualisation and analysis with virtual observatory tools, such as TopCat. During the science preparation phase (2009-2015), the CARMENES science working group also had access to Carmencita through a website created to access a relational database in a server, located in Madrid (UCM), running on Mac OS X Mountain Lion 10.8.5 with MYSQL 5.6. The website consisted of a collection of HTML and PHP 6.0 codes that recovered data from the original ascii input catalogue, and allowed basic searches (by coordinates, spectral types, magnitudes and previous observations). 


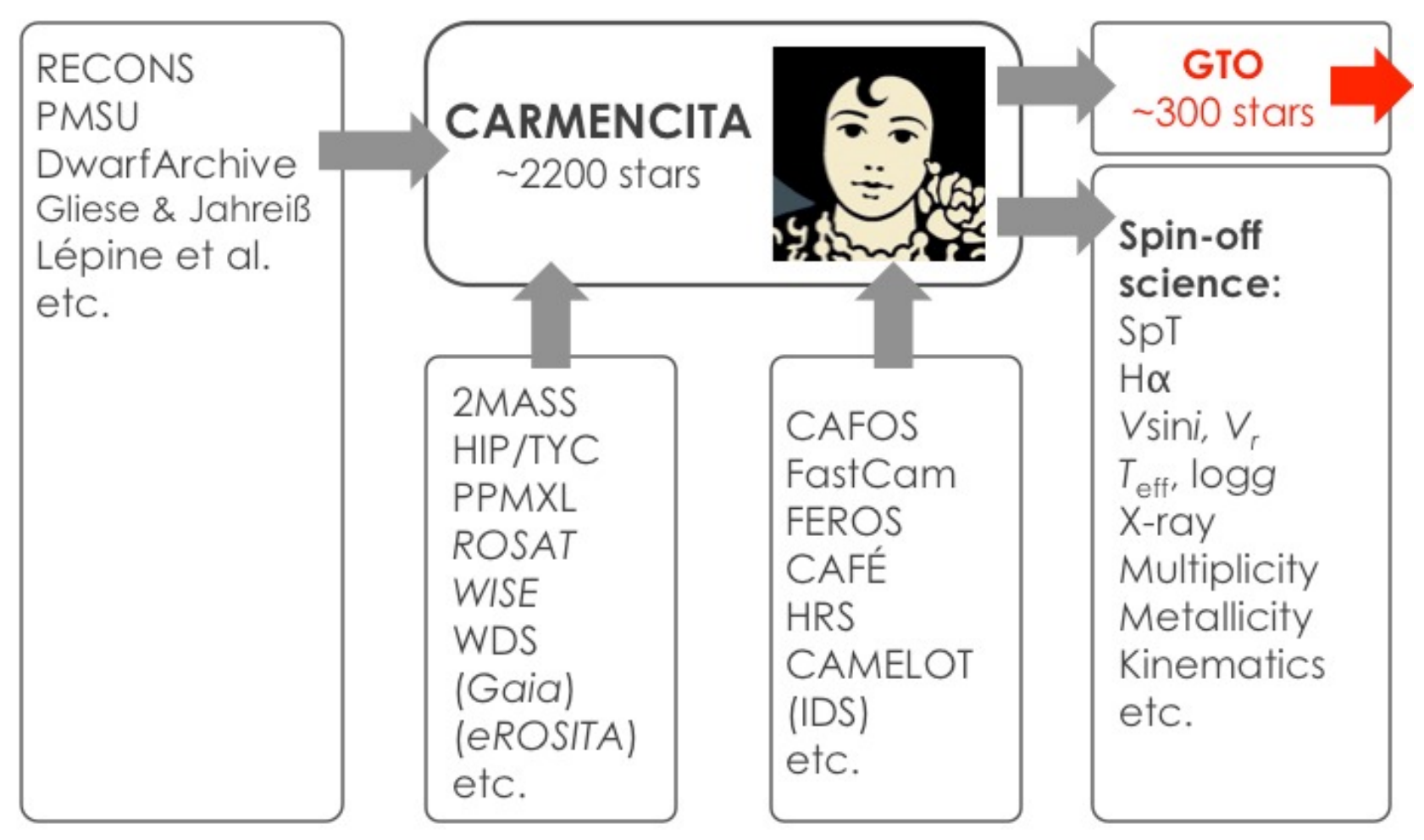

Figure 2: CARMENES data flow. I. Input catalogue (Carmencita). The data flow continues in Fig. 3.

The private catalogue, including preparatory science observations, is being made public as a CARMENES legacy through VizieR and a spin-off series of refereed papers on activity, multiplicity, basic astrophysical stellar parameters and kinematics (e.g., Alonso-Floriano et al. 2015; Cortés-Contreras et al. 2016).

The approximately 300 brightest Carmencita $M$ dwarfs for each spectral subtype without any companion at less than 5 arcsec, either bound or unbound, feed the CARMENES scheduler.

\subsection{Scheduler, Instrument Control System and Graphical User Interface}

The CA[RMENES] Scheduling Tool, CAST, takes into account observational constraints and distributes the available telescope time with genetic algorithms amongst the $\sim 300$ targets of the GTO survey. CAST provides the observer, through the Instrument Control System (ICS) Graphical User Interface (GUI), with the best M dwarf to be monitored next. The CAST main parameters are visibility, elevation, Moon phase and separation, dome and telescope variables, weather, and the most basic star parameters (coordinates, spectral type, magnitude[s], weights assigned by the Project Scientist). The CAST goals are to minimise the overhead time and maximise the exoplanet yield (García-Piquer et al. 2014, 2016). The scheduler is only available for GTO observations. 


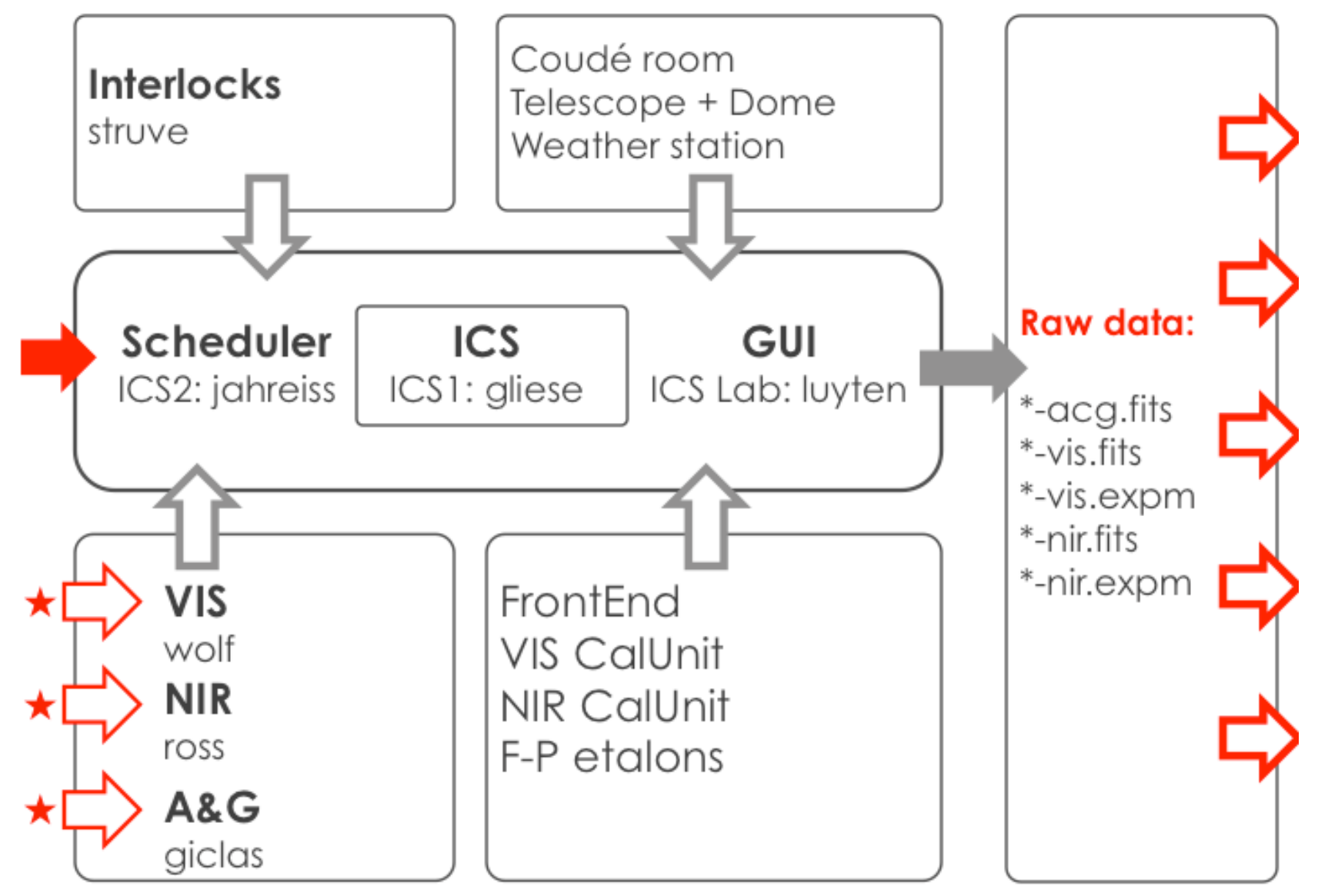

Figure 3: CARMENES data flow. II. Scheduler, Instrument Control System, Graphical User Interface, raw data and FITS headers. The data flow continues in Fig. 5.

The CARMENES ICS, which coordinates and manages the operations of the different subsystems, is described in detail by Colomé et al. (9913-149). In short, the ICS monitors and commands the computers of the VIS and NIR channels and the acquisition and guiding system (Table 1), and the controllers of the front-end, VIS and NIR calibration units and Fabry-Pérot etalons, interfaces with the telescope and dome, and passively receives data from the interlocks computer, coudé room and observatory weather station (Fig. 3). The heart of the system is composed of three computers: "ICS One", which runs the ICS and interacts with all the subsystems, "ICS Two", which runs the scheduler, and "ICS Lab", which runs the GUI. The two formers have FTP servers and can run the ICS and scheduler in case of fail of one of the computers, while the latter is the interface with the observer in the remote observing room. The ICS logs the full parameter status of all the CARMENES subsystems with an interval of frequencies that range to less than one minute for the weather station, to one second for the exposure meters.

The GUI is designed to be as simple and friendly as possible, for easy using by any observer (Fig. 4). Since early April 2016 (three months after the survey start), night observations are usually carried out in service mode by technical staff of the Department of Astrophysics of the Calar Alto observatory, with frequent (on-site and on-line) support from experienced individuals of the consortium. Given the small number of movable parts and fixed instrument configurations, observing with CARMENES requires a very short training. 


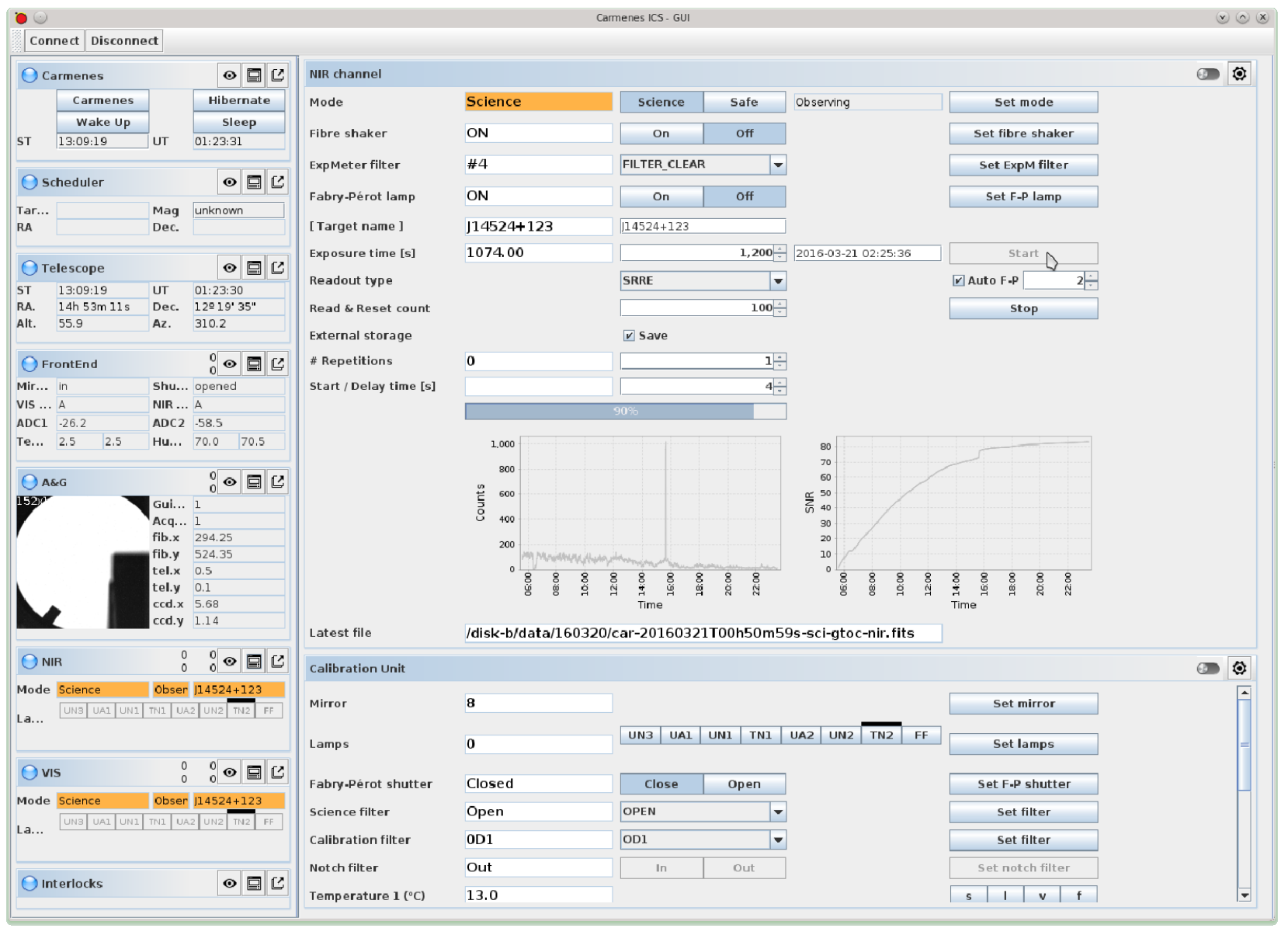

Figure 4: A capture of the CARMENES ICS GUI (example: NIR channel tab). At the moment of writing these lines, we are introducing tiny changes in the appearance and functionalities of the GUI, such as the control of two new Fabry-Pérot shutters installed in June 2016 or the option of executing observing blocks limited by signal-to-noise ratio instead of by exposure times.

\subsection{Raw data and FITS headers}

The output of an observing block (an "exposure") is a set of five files per target and epoch of observation: acquisition image (*-acg.fits), VIS spectrum (*-vis.fits), VIS exposure-meter count rate (*-vis.expm), NIR spectra (*-nir.fits) and NIR exposure-meter count rate (*-nir.expm). The channel exposure meters consist each of an off-axis parabollic mirror that collects the light of the zeroth order of the respective échelle grating, a fibre that carries that light to the channel rack, and a (visible or near-infrared) photo-multiplier. The exposure-meter files are ascii files containing time, flux and position of the exposure-meter filter. The VIS and NIR photo-multipliers are read every second.

All output files share a common string such as car-yymmddThh:mm:ss-Xxx-yyYY-*. It indicates the instrument (CARMENES), year, month and day, hour, minute and second of start of observing block in Universal Time, image type (sci: science, cal: calibration, tst: test), and four-character programme code as defined by the observatory (in open time: three first characters of surname and first character of name, e.g. cabj for the first author of this contribution). The three FITS files have comprehensive headers provided by all subsystems. We provide the exhaustive example of the template of FITS headers for the VIS channel as an appendix at the end of this contribution. 


\subsection{First pipeline (CARACAL)}

During standard operation at night, CARMENES simultaneously gets light from a target in the first (science) fibre and the corresponding Fabry-Pérot etalon in the second (calibration) fibre. However, it can instead get light from the sky in the second fibre when the target is faint, or light from U-Ne, Th-Ne and U-Ar hollow cathode lamps or a flat-field halogen lamp in one or two fibres for calibration.

A few seconds after the end of the observing block and spectra readout, CARACAL (CA[RMENES] Reduction And CALibration software; Zechmeister et al. 2015) automatically makes the dark/bias correction, order tracing, flat-relative optimal extraction (FOX; Zechmeister et al. 2014) and wavelength calibration (Bauer et al. 2015) of the VIS and NIR spectra, and generates fully reduced, wavelength-calibrated 1D spectra. CARACAL also provides a rough estimate of the target radial velocity based on a comparison with a high-resolution synthetic stellar model of low effective temperature and main-sequence gravity and a series of quality-control parameters for each night (e.g., CCD readout noise and gain, mean flux of the calibration lamp spectra, median resolution, absolute radial-velocity drift). Recently, we have implemented a new functionality that makes a coarse instrument-response correction and merges all the orders in a single matrix containing wavelength, flux, error in flux and background values (useable for science cases in which ultra-precise radial velocities are not necessary). In the case of the NIR channel only, there is an intermediate stage, run in the NIR computer, that pre-processes the individual GEIRS frames obtained in sample-up-the-ramp mode and generates a single frame that can be read by CARACAL.

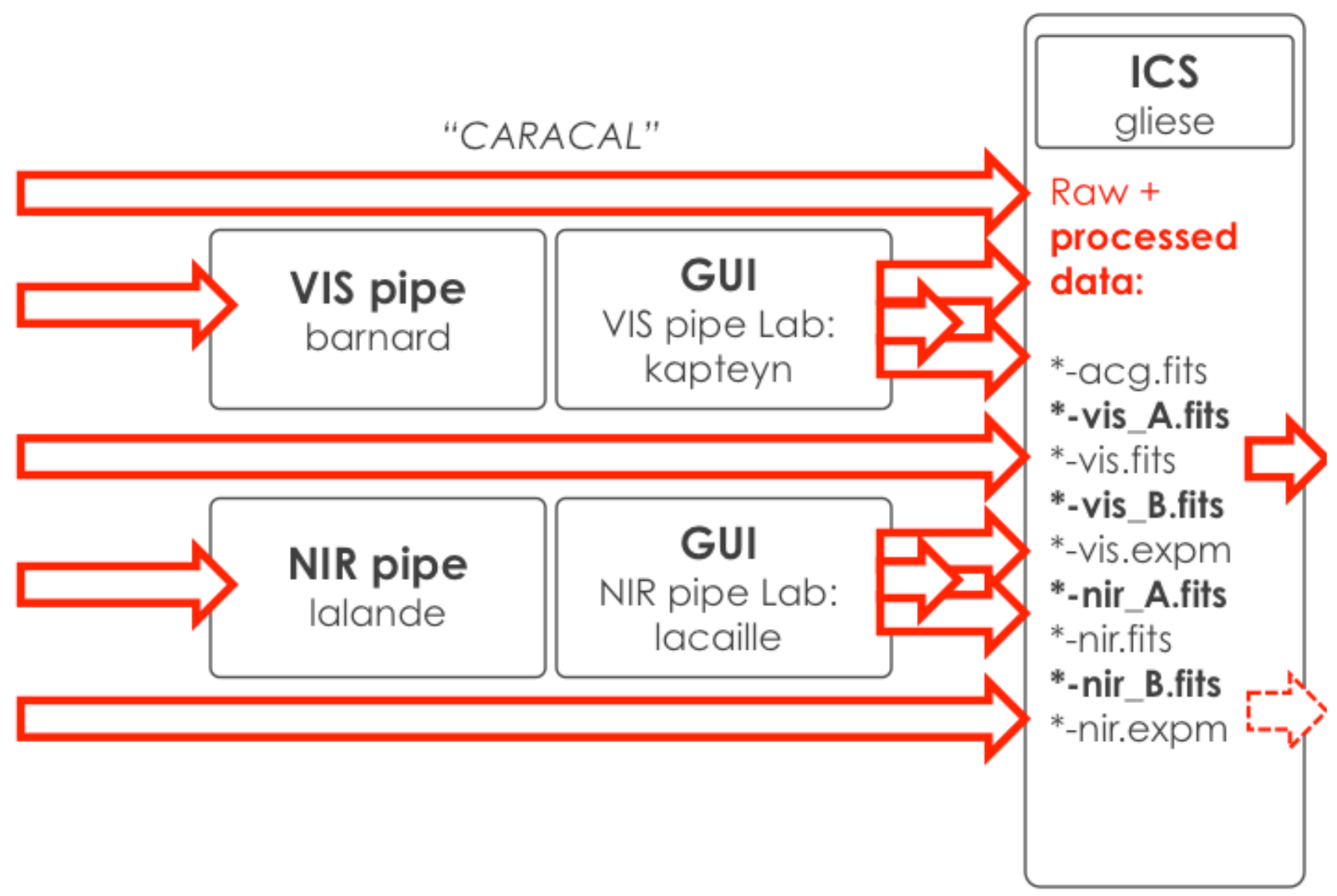

Figure 5: CARMENES data flow. III. First pipeline (CARACAL). The data flow continues in Fig. 7. 
The basis of CARACAL was the IDL REDUCE package developed by Piskunov \& Valenti (2002), to which many scripts have been added or some parts modified. The CARACAL package uses IDL v6.3, inotify, xterm, ds9, gnuplot, Tk/Tcl 8.5, git and, for miscellaneous tasks, latex2html, gps, imhead and ech2dat. We have two dedicated servers for the spectra extraction, one for each channel ("VIS pipe" and "NIR pipe" in Fig. 5); as in the case of the ICS computers, one server can run the two extraction pipelines simultaneously. We have another two desktops ("VIS pipe Lab" and "NIR pipe Lab" in Fig. 5), which are located near the the ICS Lab one with the ICS GUI, run the pipeline GUIs (dubbed xpipe) and display the extracted spectra in real time.

In trigger mode, CARACAL waits for new raw files that are provided by the ICS and located in the nigh folder path /disk-b/data/yymmdd/ in ICS One (or ICS Two). The raw FITS files (science and calibration spectra) are automatically processed by CARACAL but are not modified. From the exposure-meter files, CARACAL computes the effective mean time of observation - flux weighted. All information needed for the data reduction is passed via FITS header keywords (e.g., calibration type).

The calibration and data reduction products are stored in separate directories in the pipeline servers. For each observing block, CARACAL generates four processed files, two for each channel, and one for each of the two fibres in the field of view of the acquisition and guiding system (science and calibration). The processed data are copied back to the same night directory in the ICS One computer, were we temporarily store the nine files (five raw, four processed) per observing block.

\subsection{Observatory repository (CAHA Archive)}

In the following morning, all files stored in the previous night folder are copied to a repository common to all instruments at the CAHA Observatory. In this proceeding, we use the name "CAHA Archive" for this repository, but it should not be confused with the "SVO Calar Alto Archive", which contains both raw and science-ready public data (after proprietary time), was designed in compliance with the standards defined by the International Virtual Observatory Alliance, was built and is maintained by the Spanish Virtual Observatory, and is located in Madrid ${ }^{2}$ (Solano et al. 2012).

The current CAHA Archive is an evolution of a system initiated in 2010, which was designed under the principles of flexibility, reliability, robustness, security access, data storage, and hardware and software modularity and scalability. In the last years, the CAHA Archive has been deeply modified for optimising and adapting it to the requirements and features of the CARMENES operations. The CAHA Archive provides a large centralised storage capacity, data validation and back-up, nomenclature unification and a system for distributing the generated data to the principal investigator of the corresponding programme.

The three main hardware elements of the CAHA Archive are:

- Server. Dell PowerEdge R520 with two processors Intel Xeon E5-2407 2.40GHz Quad-Core, 32GB DDR3 RAM, a PERC 810 RAID controller with two 146GB 15K SAS discs configured as RAID1, and two hot-plug redundant power supplies.

- Discs array. Dell PowerVault MD1000 with $15 \times 2$ TB SATA disks (effectively 24TB RAID), and redundant power supply. The discs array is connected to the server through a SAS Host Bus Adapter cable and Dell SAS 5/E PCIe HBA Controller Cards.

- Network connection. Two $1 \mathrm{~Gb} / \mathrm{s}$ net interfaces in bonding mode, which provide high-speed access and avoid bandwidth problems due to multiple server access.

We plan to periodically increase the storage capacity of the CAHA Archive. For guaranteeing enough capacity for the expected long operations lifetime of CARMENES, the next extension will contain another storage array of about $90 \mathrm{~TB}$.

Over this hardware it is running a customised software developed with Python 2.6 and based on a MYSQL 5.0 database, pyfits 3.3 libraries and SUSE Linux Enterprise Server 11 (x86_64). The interactive side of this system uses Apache 2.2 and PHP5 to provide a web interface to the operator for manual interaction in the open/capture/classify/close process. There is also an FTP server for making data accesible to the principal investigator.

${ }^{2}$ http: //caha.sdc.cab.inta-csic.es/calto/ 
The core of CAHA Archive operations is a Python daemon that waits for a trigger for starting capturing observing block data defined by date/path/instrument/telescope. First, for CARMENES, the daemon determines which of the two ICS servers is active for data capturing (generally, ICS One). Next, it generates a thread for getting, storing, classifying and distributing all the FITS spectra (raw and reduced) and attached files (A\&G image, exposure-meter files) found in the path of the active server.

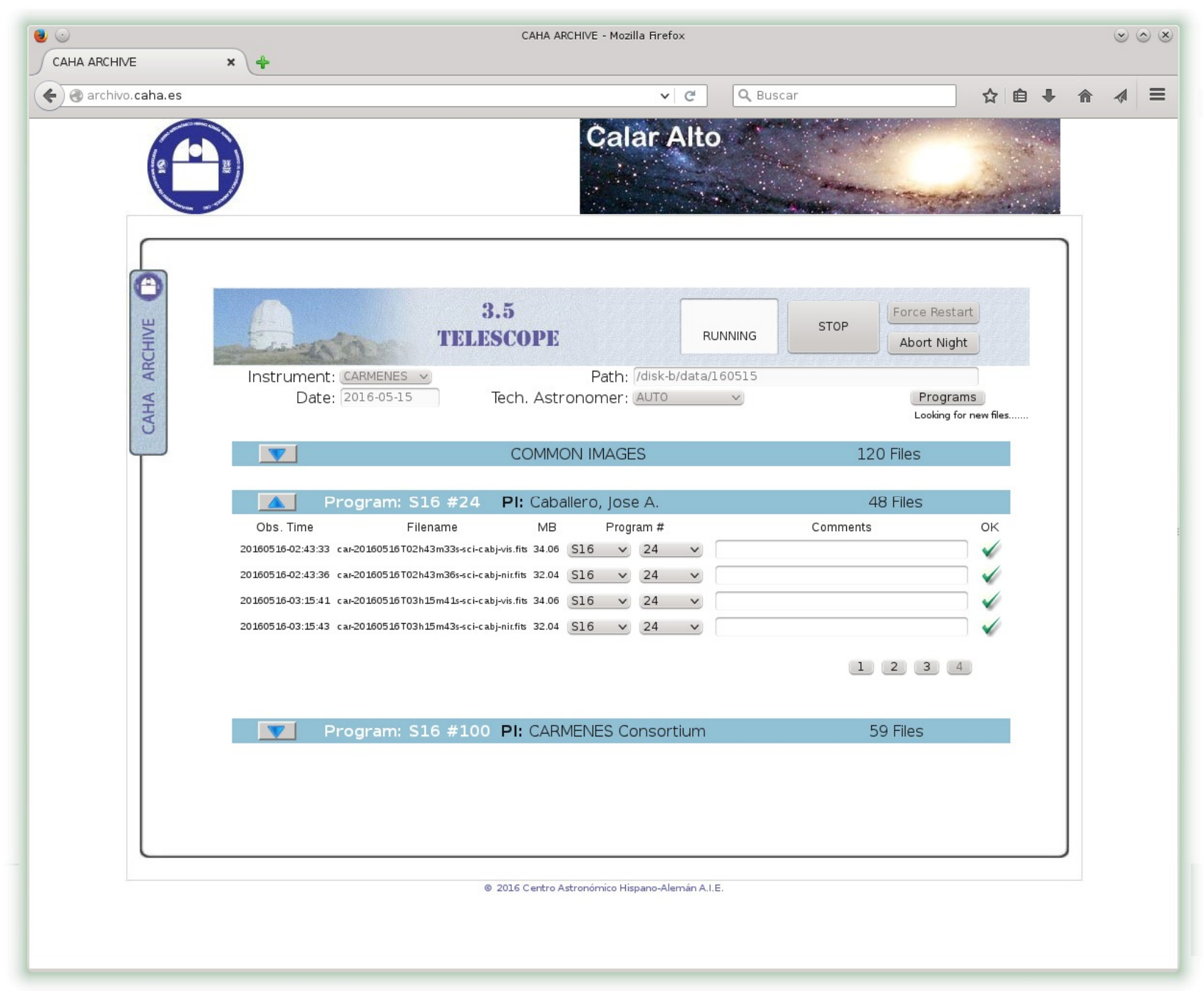

Figure 6: Operational view of a running CAHA Archive process.

The trigger is set automatically at 08:00 UT of the next morning (if it has not been started manually earlier). Although the CAHA Archive can be running during night observations and get the files as soon as they are written in the folder, the automatic trigger set avoids the CAHA Archive server to get slow when the bandwith is more necessary (at night). The possibility of manually starting the capture and making a classification of spectra is available to the night observer through a secure website (Fig. 6). This possibility will provide flexibility for adapting to errors or special situations. When one of these threads is activated, it starts a cyclic procedure by which the CAHA Archive:

- Looks for new files in the path of the night folder (the same as for CARACAL: /disk-b/data/yymmdd/). 
- Discards images saved previously.

- Downloads only new files using FTP.

- Checks files and gets necessary headers necessary for archiving: NAXIS, NAXIS1, NAXIS2, FILENAME, OBJECT, DATE-OBS, EXPTIME, RA, DEC, EQUINOX, PROG-NUM, PROG-PI, OBSERVER and HIERARCH CAHA INS ICS IMAGETYP. The system does not try to fix header keywords or rename FITS files (during commissioning and early phases of science surveys, a few bugs in the data flow were still present).

- Stores new FITS files and registers them in the MySQL database,

- Classifies the FITS files and assigns them automatically to its observation program. The ascii exposure-meter files are associated to the FITS files based on the common part of the file name. All the daily calibration files are assigned by default to the "Common programme", which is the way to assign files to all observation programmes executed on the same night. Another special option called "No program associated" is used when the system cannot classify automatically a file.

The length of each cyclic procedure depends on the number and size of the files generated per night, ranging from a few minutes to more than half an hour. At the end of the procedure, it checks if a "close archive" flag is active. When the procedure was started autonomously, this flag is set automatically $30 \mathrm{~min}$ after receiving the last file (the flag will also be able to be set manually using the web interface). If the flag is inactive, the cyclic procedure goes on.

When the "close archive" flag is active, the system identifies the observation programmes developed that night, sends by automatic email to the corresponding principal investigator with an FTP user account, a password and the instructions for retrieving the data (only on the first night that data were collected for his/her programme), and connects to the Calar Alto observatory FTP server (ftp.caha.es). With his/her password, the principal investigator can easily download the data sorted by night folders in his/her personal area in the FTP. The CAHA Archive manages automatically the FTP server by creating/announcing/destroying accounts and distributing the corresponding data.

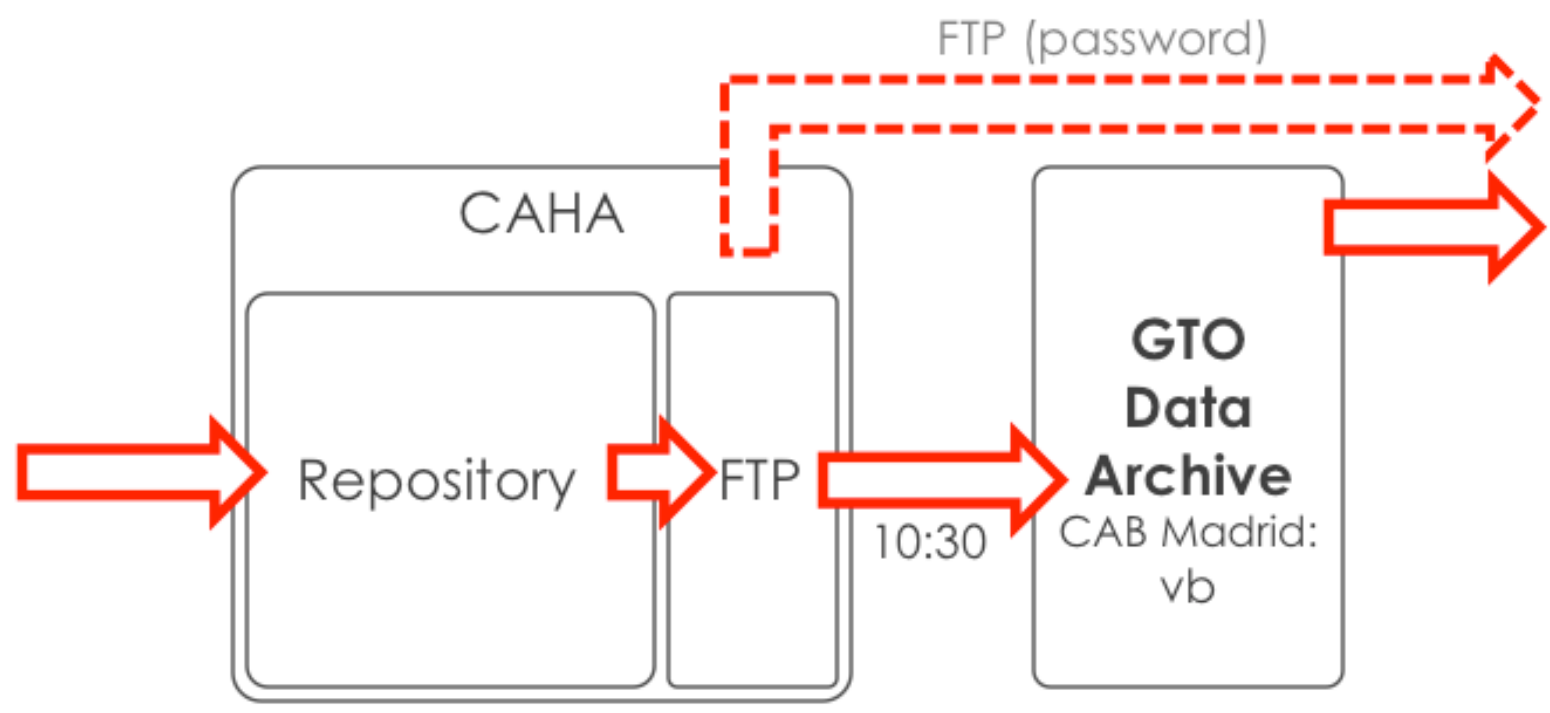

SSH/vncviewer (barnard/lalande)

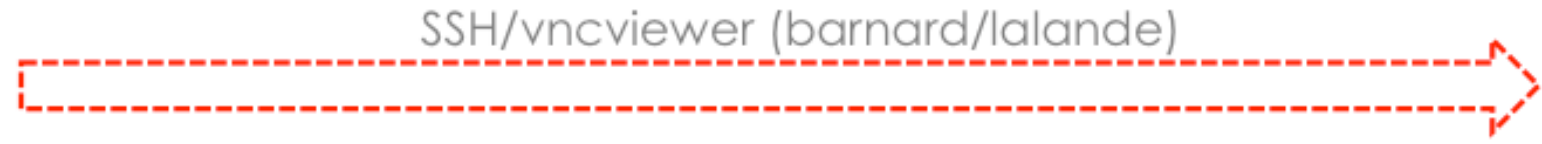

Figure 7: CARMENES data flow. IV. CAHA Archive (observatory repository) and Guaranteed Time Observations Data Archive. The data flow continues in Fig. 9. 
The described archiving, triggering and data retrieval by FTP is common to guaranteed, open and director discretional time observations with CARMENES. However, there are some differences in the GTO data flow starting from this point. First, only the principal investigator of the open and director discretional time observations receives in the morning of every observed night an automatic email with the status report of his/her programme (this email is independent of the CAHA Archive). For GTO data, we implemented a synchronisation process between the CAHA Archive in Calar Alto and the GTO Data Archive in Madrid, which is automatically triggered at 10:30 UT every day. Since CARMENES is scheduled for about $90 \%$ of the available time of the $3.5 \mathrm{~m}$ Zeiss telescope, and we take calibration sequences in the afternoon even when CARMENES is not scheduled, the synchronisation is executed seven days per week, 365 days per year.

Open and director discretionary time data will be made public after one year of proprietary time through the "SVO Calar Alto Archive" (cf. Solano et al. 2012). The propietary time of GTO data is up to three years. National guaranteed time data may never be public.

After five months since the start of the CARMENES science operations on 2016 Jan 01, the CAHA Archive has digested more than 25,000 raw FITS files (*_acg.fits, *_vis.fits and *_nir.fits), 17,000 reduced FITS spectra and 25,000 exposure-meter files, amounting to about $900 \mathrm{~GB}$ of data corresponding to five different CARMENES programmes (GTO, three open time, one director-discretionary time).

For speeding up the process and solving a problem of the NIR and/or VIS pipelines not running automatically every night, we implemented a third way of retrieving CARMENES data, only feasible by SSH or vncviewer by the manager of the pipeline computers in Göttingen. Once all the data are homogenously re-processed with the latest version of the CARACAL pipeline, the reduced data are then sent by FTP from Göttingen (IAG) to the GTO Data Archive in Madrid (CAB), which makes them available to all the members of the CARMENES Consortium.

\subsection{Guaranteed Time Observations Data Archive}

The CARMENES GTO Data Archive ${ }^{3}$, hosted by the Spanish Virtual Observatory in Madrid (CAB), provides easy and reliable access to raw and processed data obtained during guaranteed time observations. This provision is through a friendly web browser interface (Fig. 8). The core of the GTO Data Archive is a server developed with free tools, mostly Java, Hibernate and PostgreSql, running on a desktop ${ }^{4}$ with Ubuntu 14.04.1 LTS. It provides a login mechanism with three access levels (public data, consortium, administrator) and the capability to search data by target name (Carmencita identifier; Quirrenbach et al. 2015) or by range of nights. The system automatically analyses and shows only the available data sorted by channel (VIS, NIR), type (calibration, science) and/or processing completion (raw, reduced). FITS headers are available for all users in ascii format. Data can be downloaded as FITS files (single files) or as a zip file (multiple files).

\subsection{Second pipeline (SERVAL)}

An exclusive GTO step in the CARMENES data flow, SERVAL is a second pipeline that runs in Göttingen (IAG) and computes series of ultra-precise radial-velocity measurements of the $\sim 300$ monitored $\mathrm{M}$ dwarfs via least-square fit, template co-adding, telluric masking and proper échelle order weighting. SERVAL also measures differential full-width half maxima, chromatic indicators and the pseudo-equivalent widths of $\mathrm{H} \alpha \lambda 6562.8 \AA$. It has the power to implement new modules that measure other spectral activity indicators (indices of the calcium triplet and sodium doublet, emission lines in the near infrared, rotational velocities). It will be described in detail by Zechmeister et al. (in prep.).

\footnotetext{
${ }^{3}$ http: / / carmenes. cab.inta-csic.es

${ }^{4}$ It is named vb after G.-A. van Biesbroeck (1880).
} 

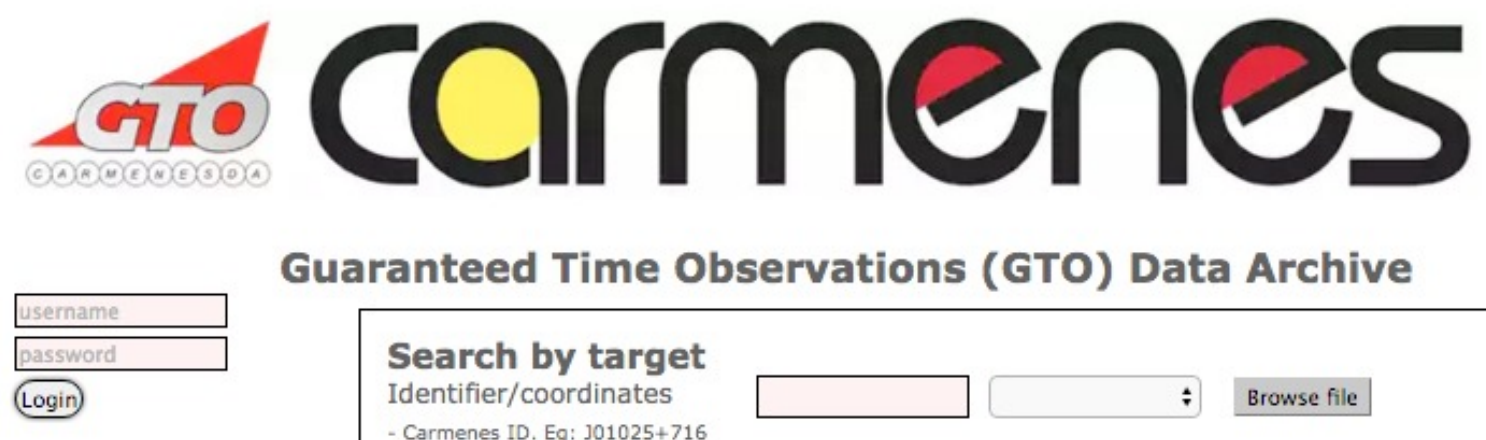

\section{Guaranteed Time Observations (GTO) Data Archive}
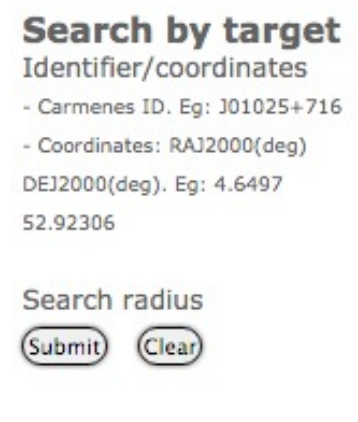

\section{Search by Date}

Date from $\square$ to

Category? Sci $\square$ Cal $\square$ Acg $\square$

Submit Clear

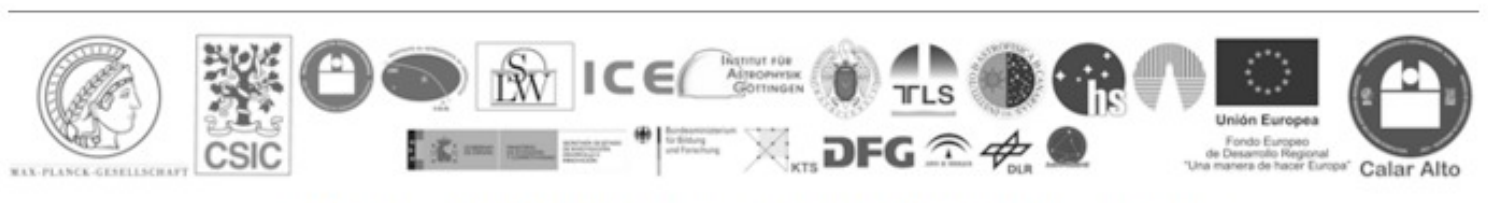

(C) 2009-2016 | CARMENES Consortium | Spanish Virtual Observatory | Help desk

Figure 8: Capture of the home webpage of the CARMENES GTO Data Archive.

\subsection{Radial-velocity data archive (RADAR) and visualisation tool (CAVEMAN)}

The output of SERVAL is injected into RADAR, the RAdial velocity Data ARchive, which can be visualised by CAVEMAN, the CA[RMENES] Visualisation, Evaluation and Multi-Analysis Notebooks. Both the RADAR archive and the CAVEMAN code repository are developed in Heidelberg (MPIA) and based on Apache Subversion ${ }^{5}$. Besides, CAVEMAN makes use of Jupyter Notebook $^{6}$ to provide data visualisation and analysis routines. RADAR and CAVEMAN are web-based tools designed for storing and working with data generated not only by SERVAL, but also by the leaders of some internal CARMENES science work packages (Fig. 9). While RADAR is mostly a database, CAVEMAN uses the data products stored by RADAR and provides users with standard and customisable sets of visualisation and analysis procedures with the final aim of detecting and characterising radial-velocity exoplanet signals, and disentangling them from stellar activity. Closing the loop of data flow, RADAR and CAVEMAN need some of the parameters originally tabulated by Carmencita.

\footnotetext{
${ }^{5}$ http: // subversion. apache.org

${ }^{6}$ http: //jupyter.org
} 


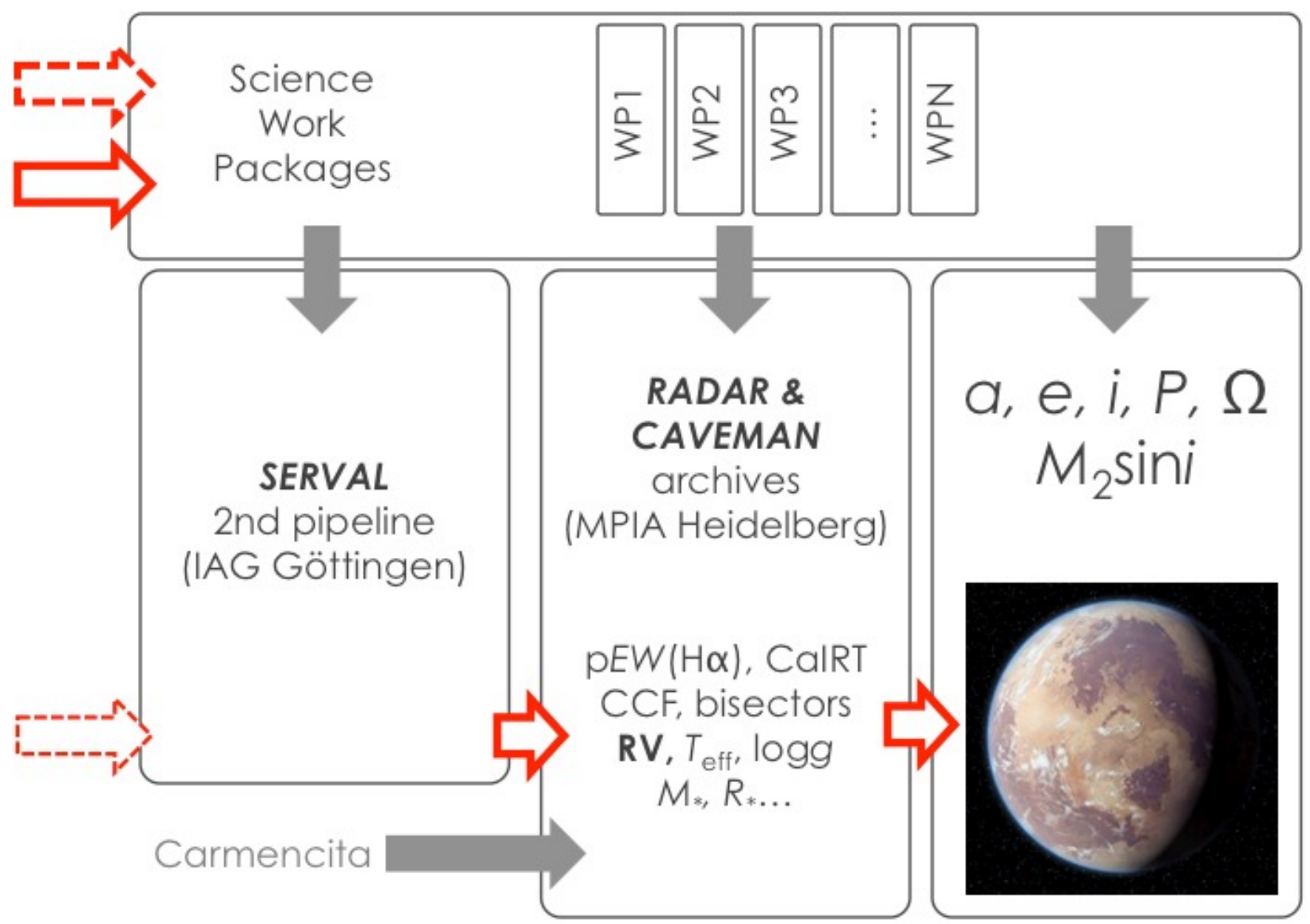

Figure 9: CARMENES data flow. V. Second pipeline, radial-velocity archive, visualisation tool and final planetary system parameters.

The scope of CAVEMAN is threefold. First, it will be used to generate a standard analysis report for every star of the CARMENES survey. Second, it will provide an extendable standard set of data visualisation and analysis tools for the users of the survey data. These python-based tools are very flexible and can be modified by all users according to their needs. Third, CAVEMAN will provide a platform for the joint development and contribution of visualisation and analysis tools by the CARMENES user community. Such contributed tools can in principle become standard tools. This will make it possible to bring the expertise from different groups together, thereby optimising the scientific analysis of every star of the survey.

\section{SUMMARY}

CARMENES is the first astronomical instrument that delivers spectra covering from $0.5 \mu \mathrm{m}$ to $1.7 \mu \mathrm{m}$ with spectral resolution $\mathrm{R}>80,000$ and long-term stability of $\sim 1 \mathrm{~m} \mathrm{~s}^{-1}$. Because of that, it is becoming a cornerstone for the search and confirmation of very low-mass exoplanets (mini-neptunes, super-earths and less massive ones) around the coolest and most abundant stars of the Milky Way. For reaching the required stability, the CARMENES data flow is exquisitely tracked, monitored and analysed from the target selection, through the spectra compilation, to the data reduction and archiving. 


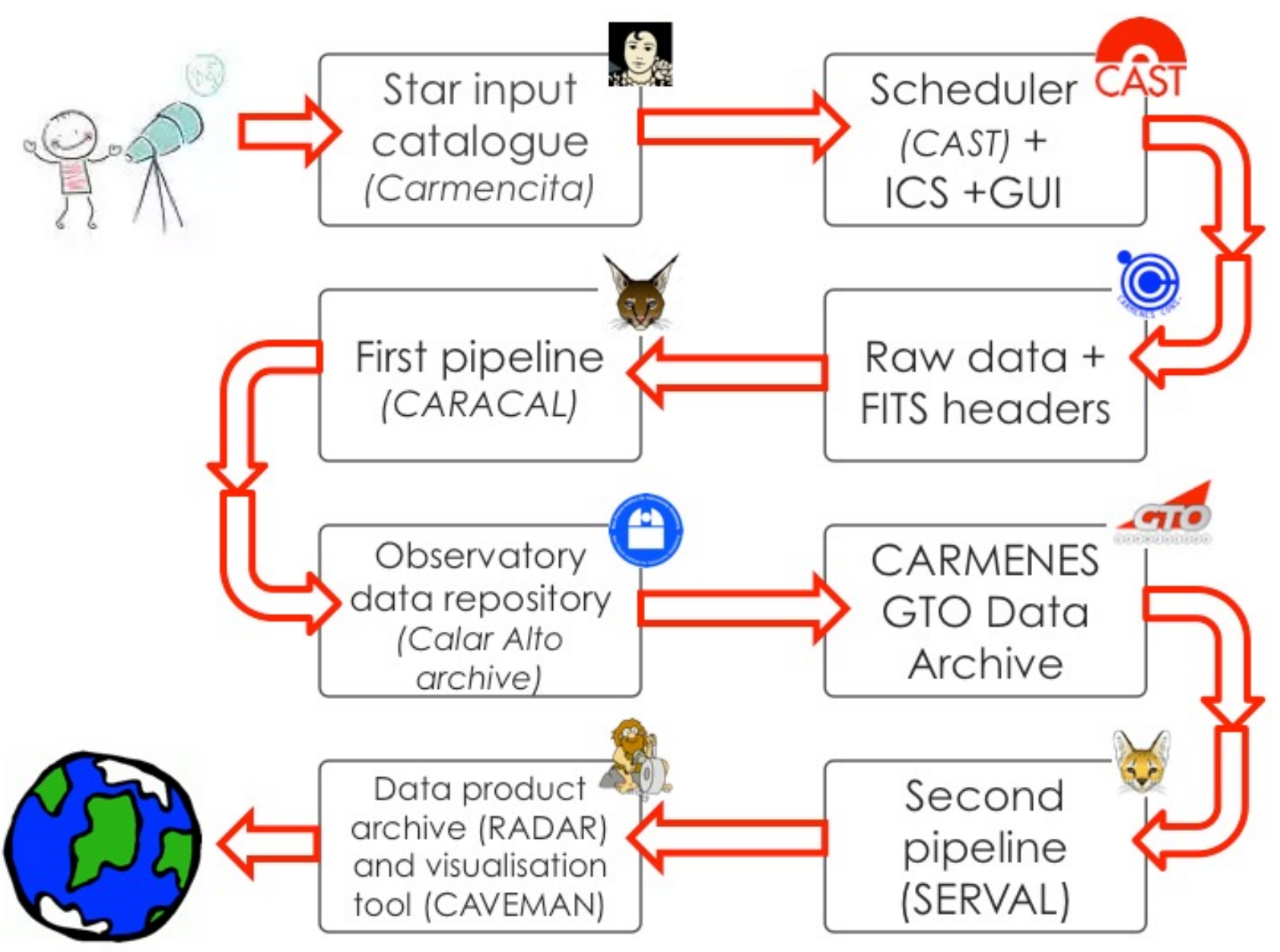

Figure 10: The whole CARMENES data flow from the astronomer to the exoearth.

In this proceeding, we describe in detail all the steps of the CARMENES guaranteed data observations data flow (Fig. 10): (i) the CARMENES input catalogue (Carmencita); (ii) the scheduler (CAST), instrument control system and graphical user interface; (iii) raw data and FITS headers; (iv) the spectrum extraction and wavelength-calibration pipeline (CARACAL); ( v) the observatory data repository and archive; (vi) the guaranteed time observations data archive; (vii) the radial-velocity pipeline (SERVAL); (viii) the data products archive (RADAR) and visualisation tool (CAVEMAN).

\section{ACKNOWLEDGEMENTS}

CARMENES is an instrument for the Centro Astronómico Hispano-Alemán de Calar Alto (CAHA). CARMENES was funded by the German Max-Planck-Gesellschaft (MPG), the Spanish Consejo Superior de Investigaciones Científicas (CSIC), the European Union through European Regional Fund (FEDER/ERF), Spanish Ministry of Economy and Competitiveness, the state of Baden-Württemberg, the German Science Foundation (DFG), the Junta de Andalucía, and by the Klaus Tschira Stiftung, with additional contributions by the members of the CARMENES Consortium (MaxPlanck-Institut für Astronomie, Instituto de Astrofísica de Andalucía, Landessternwarte Königstuhl, Institut de Ciències de l'Espai, Institut für Astrophysik Göttingen, Universidad Complutense de Madrid, Thüringer Landessternwarte Tautenburg, Instituto de Astrofísica de Canarias, Hamburger Sternwarte, Centro de Astrobiología, and the Centro Astronómico Hispano- Alemán). We thank Richard Mathar for his exhaustive comments and suggestions. 


\section{REFERENCES}

1. Alonso-Floriano, F. J., Morales, J. C., Caballero, J. A. et al., "CARMENES input catalogue of M dwarfs. I. Lowresolution spectroscopy with CAFOS", A\&A, 577, A128 (2015).

2. Bauer, F. F., Zechmeister, M., Reiners, A., "Calibrating echelle spectrographs with Fabry-Pérot etalons", A\&A, 581, A117 (2015).

3. Becerril, S. et al., "CARMENES: how to achieve the full AIV at system level of a cryo-instrument in nine months", this SPIE, 9908-259 (2016)

4. Bidelman, W. P., "G. P. Kuiper's spectral classifications of proper-motion stars", ApJS, 59, 197 (1985)

5. Caballero, J. A., Cortés-Contreras, M., Alonso-Floriano, F. J. et al., "CARMENES at PPVI. CARMENCITA Herbs and Spices to Help you Prepare a Genuine Target Sample", Protostars and Planets VI, 15-20 July 2013, Heidelberg, poster \#2K020 (2013).

6. Colomé, J. et al., "CARMENES: instrument control software suite", this SPIE, 9913-149 (2016)

7. Cortés-Contreras, M., Béjar, V. J. S., Caballero, J. A. et al., "CARMENES input catalogue of M dwarfs. II. Highresolution imaging with FastCam" A\&A, submitted (2016).

8. García-Piquer, Á., Guàrdia, J., Colomé, J. et al., "CARMENES instrument control system and operational scheduler", Proc. SPIE 9152, E21 (2014).

9. García-Piquer et al., "Efficient scheduling of astronomical observations. Application to the CARMENES radial velocity survey", A\&A, submitted (2016).

10. García-Vargas, M. L. et al., "CARMENES: management of a schedule-driven project”, this SPIE, 9911-24 (2016).

11. Helmling, J. et al., "CARMENES: interlocks or the importance of process visualization and system diagnostics in complex astronomical instruments", this SPIE, 9908-237 (2016).

12. Henry, T. J, Kirkpatrick, J. D. \& Simons, D. A. "The solar neighborhood, 1: Standard spectral types (K5-M8) for northern dwarfs within eight parsecs", AJ, 108, 1437 (1994).

13. Joy, A. H. \& Abt, H. A., "Spectral Types of M Dwarf Stars", ApJS, 28, 1 (1974).

14. Lee, S.-G., "Spectral classification of high-proper-motion stars", AJ, 809, 702 (1984).

15. Lépine, S. \& Gaidos, E., "An All-sky Catalog of Bright M Dwarfs", AJ, 142, 138 (2011).

16. Lépine, S., Hilton, E. J., Mann, A. W. et al., "A Spectroscopic Catalog of the Brightest $(J<9)$ M Dwarfs in the Northern Sky", AJ, 145, 102 (2013).

17. Mason, B. et al., "The 2001 US Naval Observatory Double Star CD-ROM. I. The Washington Double Star Catalog", AJ, 122, 3466 (2001).

18. Pérez-Calpena, A. et al., "CARMENES: system engineering during manufacturing and AIV phases", this SPIE991178 (2016).

19. Piskunov, N. E. \& Valenti, J. A., "New algorithms for reducing cross-dispersed echelle spectra", A\&A, 385, 1095 (2002).

20. Quirrenbach, A., Amado, P. J., Mandel, H. et al., "CARMENES: Calar Alto high-Resolution search for M dwarfs with Exoearths with a Near-infrared Echelle Spectrograph", Proc. SPIE 7735, E37 (2010).

21. Quirrenbach, A., Amado, P. J., Seifert, W. et al., "CARMENES instrument and survey overview", Proc. SPIE, 8446, E0R (2012).

22. Quirrenbach, A., Amado, P. J., Caballero, J. A. et al., "CARMENES instrument overview", Proc. SPIE 9147, E1F (2014).

23. Quirrenbach, A., Caballero, J. A., Amado, P. J. et al., "Manufacturing, Assembly, Integration and Verification of CARMENES and Preparation of its Input Catalogue", 18th Cambridge Workshop on Cool Stars, Stellar Systems, and the Sun, 8-14 Jun 2014, Lowell Observatory, USA, 897 (2015).

24. Quirrenbach, A., Amado, P. J., Caballero, J. A. et al., "CARMENES: an overview six months after first light", Proc. SPIE 9908, this volume

25. Reid, I. N. Hawley, S. L. \& Gizis, J. E. et al., "The Palomar/MSU Nearby-Star Spectroscopic Survey. I. The Northern M Dwarfs -Bandstrengths and Kinematics", AJ, 110, 1838 (1995).

26. Roeser, S., Demleitner, M. \& Schilbach, E., "The PPMXL Catalog of Positions and Proper Motions on the ICRS. Combining USNO-B1.0 and the Two Micron All Sky Survey (2MASS)", AJ, 139, 2440 (2010)

27. Scholz, R.-D., Lehmann, I., Matute, I. and Zinnecker, H. "The nearest cool white dwarf (d $\sim 4$ pc), the coolest Mtype subdwarf (sdM9.5), and other high proper motion discoveries", A\&A, 425, 519 (2004).

28. Seifert, W. et al., "CARMENES: VIS channel spectrograph", this SPIE, 9908-231 (2016). 
29. Zechmeister, M., Anglada-Escudé, G., Reiners, A., "Flat-relative optimal extraction. A quick and efficient algorithm for stabilised spectrographs", A\&A, 561, A59 (2014).

30. Zechmeister, M., Zhao, J., Marvin, C. et al., "CARACAL - The CARMENES pipeline", Extreme Precision Radial Velocity workshop, 5-8 July 2015, Yale University, USA, published on-line (2015).

\title{
APPENDIX: VIS-CHANNEL FITS HEADER TEMPLATE
}

\author{
SIMPLE = T/ File conforms to FITS standard \\ BITPIX = 16 / Number of bits per data pixel \\ NAXIS = 2 / Number of data axes \\ NAXIS1 = 4250 / Length of data axis 1 \\ NAXIS2 = 4250 / Length of data axis 2 \\ COMMENT FITS (Flexible Image Transport System) 2010A\&A...524..A42P \\ EXTEND = T / FITS dataset may contain extensions \\ BZERO = 32768 / Data value offset \\ BSCALE = 1 / Data value scale factor \\ DATAMAX = / Maximum data value in array \\ DATAMIN = / Minimum data value in array \\ FILENAME = ' ' / This file name \\ OBJECT = 11 / Observed object \\ $\mathrm{DATE}-\mathrm{OBS}=1, \quad$ / UTC at observation start \\ MJD-OBS = / Modified Julian Date at observation start \\ LST = 11 / [h] Local sidereal time \\ EXPTIME = / [s] Exposure time on VIS CCD \\ $\mathrm{RA}=$ / [deg] [hh:mm:ss.ss] Requested right ascension \\ $\mathrm{DEC}=$ / [deg] [+dd:mm:ss.s] Requested declination \\ RADESYS = 'FK5 1 / Reference frame of equatorial coordinates \\ EQUINOX $=2000.0 \quad$ / Equinox of the coordinate system \\ AIRMASS = / Airmass at observation start \\ PROG-NUM = ' ' / CAHA internal programme number \\ PROG-PI = 11 / CAHA internal programme PI identifier \\ OBSERVER = ' ' / Observer surname or identification \\ FOLDER = ' ' / Corresponding night data folder \\ OBSERVAT = 'CAHA ' / Centro Astronomico Hispano-Aleman de Calar Alto \\ TELESCOP $=1 \mathrm{CA}-3.5$ ' / $3.5 \mathrm{~m}$ Calar Alto Telescope \\ INSTRUME $=$ ' CARMENES ' / CARMENES \\ SUBSYS = 'vis / / Visual channel \\ REFERENC= '2014SPIE.9147E..1FO' / Quirrenbach et al. 2014, SPIE, 9147, E1F \\ ORIGIN = 'CARMENES' / http://carmenes.caha.es \\ COMMENT1 $=1, \quad /$ Exposure comment \\ COMMENT2 $=1, \quad$ / Night comment \\ HIERARCH CAHA INS SCHEDULER MODE = $11 /$ Scheduler mode (GTO only) \\ HIERARCH CAHA INS SCHEDULER KARMN $=1, /$ / Carmencita name (GTO only) \\ HIERARCH CAHA INS SCHEDULER RA = ' / / Carmencita RA J2000 (GTO only) \\ HIERARCH CAHA INS SCHEDULER DEC $=11$ / Carmencita DEC J2000 (GTO only) \\ HIERARCH CAHA INS SCHEDULER J MAG = / [mag] J magnitude (GTO only)

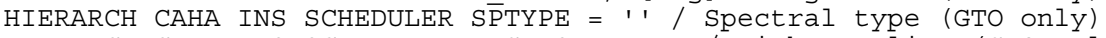 \\ HIERARCH CAHA INS SCHEDULER NIGHTQUA $=11 /$ Night quality (GTO only) \\ HIERARCH CAHA INS ICS CATEGORY $=11$ / Data category \\ HIERARCH CAHA INS ICS IMAGETYP $=1, /$ Type of observation \\ HIERARCH CAHA INS ICS FIB-MODE= 1 , / Fibre observation mode \\ HIERARCH CAHA INS ICS OBS-MODE $=11$ / ICS observing mode \\ HIERARCH CAHA INS ICS OB-NAME = 1 / Observing block name \\ HIERARCH CAHA INS ICS OB-START = '1/ UT at start of observing block \\ HIERARCH CAHA INS ICS NUM-EXP = / Cardinal number of series of exposures \\ HIERARCH CAHA INS ICS NUM-SEQ = / Ordinal number of a exposure in a series \\ HIERARCH CAHA INS ICS DATE-AVG $=1$ / UTC at midpoint of observation (ExpMeter) \\ HIERARCH CAHA INS ICS VERSION = 'VX.XX'/ Released 20160301 \\ HIERARCH CAHA GEN AMBI TEMPERATURE = / [OC] Air temperature \\ HIERARCH CAHA GEN AMBI DEWPOINT $=/$ [OC] Dew point temperature \\ HIERARCH CAHA GEN AMBI RHUM = / [\%] Relative humidity \\ HIERARCH CAHA GEN AMBI WIND SPEED $=/[\mathrm{m} / \mathrm{s}]$ Wind speed \\ HIERARCH CAHA GEN AMBI WIND GUST $=/$ [m/s] Wind gust \\ HIERARCH CAHA GEN AMBI WIND DIR = / [deg] Wind dirrection \\ HIERARCH CAHA GEN AMBI PRESSURE = / [hPa] Air pressure \\ HIERARCH CAHA GEN AMBI RAIN = / RAIN \\ HIERARCH CAHA GEN AMBI EXTINCTION = / [mag] Visual extinction in $V$ band \\ HIERARCH CAHA GEN AMBI SEEING =/ [arcsec] Seeing in V band \\ HIERARCH CAHA TEL POS SET RA =/ [deg] [hh:mm:s.s.s] Set telescope right ascension \\ HIERARCH CAHA TEL POS SET DEC = / [deg] [+dd:mm:ss.s] Set telescope declination \\ HIERARCH CAHA TEL POS SET EQUINOX = / Set telescope equinox \\ HIERARCH CAHA TEL POS EL_START = / [deg] Telescope elevation at exposure start \\ HIERARCH CAHA TEL POS AZ START = / [deg] Telescope azimuth at exposure start \\ HIERARCH CAHA TEL POS HA START = / [deg] Telescope hour angle at exposure start
}


HIERARCH CAHA TEL POS DOME_EL_UPP_E = / [deg] Upper segment limit angle HIERARCH CAHA TEL POS DOME_EL_LOW_E = / [deg] Lower segment limit angle HIERARCH CAHA TEL POS DOME $\mathrm{AZ}^{-}=/^{-}$[deg] Dome azimuth

HIERARCH CAHA TEL FOCU ID $\bar{l} /$ Cassegrain focus

HIERARCH CAHA TEL FOCU F_RATIO $=3.48 /$ FOCal ratio

HIERARCH CAHA TEL FOCU LEN $=12.195 /$ [m] FOCal length

HIERARCH CAHA TEL FOCU SCALE $=0.169 /$ [mm/arcsec] Plate scale

HIERARCH CAHA TEL FOCU VALUE $=32.988 /[\mathrm{mm}]$ Absolute telescope focus

HIERARCH CAHA TEL MIRR S1 COLLAREA $=9.09 /$ [m^2]

HIERARCH CAHA TEL GEOELEV $=2168 . /$ [m] Height above sea level

HIERARCH CAHA TEL GEOLAT $=37.2210917$ / [deg] Geographical latitude

HIERARCH CAHA TEL GEOLON $=-2.5468333 /$ [deg] Geographical longitude

HIERARCH CAHA INS FRONTEND PICKMIRR $=11 /$ Pick-up mirror position

HIERARCH CAHA INS FRONTEND ADCANG1 = / [deg] Rot-angle of 1st ADC prism

HIERARCH CAHA INS FRONTEND ADCANG2 $=/$ [deg] Rot-angle of 2nd ADC prism

HIERARCH CAHA INS FRONTEND ADCAUTO $=11 /$ Automatic set of ADC angles

HIERARCH CAHA INS FRONTEND VISCAL $=11 /$ VIS fibre cal-mirror position

HIERARCH CAHA INS FRONTEND NIRCAL $=11 /$ NIR fibre cal-mirror position

HIERARCH CAHA INS FRONTEND VISSHUTTER = / VIS channel shutter in auto mode

HIERARCH CAHA INS FRONTEND TEMPI $=/$ [OC] FE temperature near A\&G camera

HIERARCH CAHA INS FRONTEND TEMP2 $=/$ [OC] FE temperature near fibre feed

HIERARCH CAHA INS FRONTEND TEMP3 $=/$ [OC] FE temperature near elec-box

HIERARCH CAHA INS FRONTEND HUM1 $=/[\%]$ FE humidity near A\&G camera

HIERARCH CAHA INS FRONTEND HUM2 $=/[\%]$ FE humidity near fibre feed

HIERARCH CAHA INS FRONTEND GUIDE EXPTIME = / [s] Guiding individual exptime

HIERARCH CAHA INS FRONTEND GUIDE PERIOD $=/$ [s] Guiding period

HIERARCH CAHA INS FRONTEND GUIDE WEIGHT $=/$ Guiding weight

HIERARCH CAHA INS CHAMBER T-ROOM-EAST $=/[\mathrm{K}]$ NIR room temperature

HIERARCH CAHA INS CHAMBER T-FLOW-EAST $=/$ [K] NIR flow temperature

HIERARCH CAHA INS CHAMBER T-ROOM-WEST $=/$ [K] VIS roOm temperature

HIERARCH CAHA INS CHAMBER T-FLOW-WEST $=/$ [K] VIS flow temperature

HIERARCH CAHA INS CHAMBER T-ROOM-CAL $=/$ [K] Calibration room temperature

HIERARCH CAHA INS CHAMBER T-CORRIDOR $=/ / \mathrm{K}]$ Technical area temperature

HIERARCH CAHA INS VIS MODE $=11 /$ VIS channel mode

HIERARCH CAHA INS VIS FIB-SHAKER $=/$ [mA] VIS fibre shaker current

HIERARCH CAHA INS VIS EXPMETER STATUS = / VIS EXpMeter status

HIERARCH CAHA INS VIS EXPMETER FILE = 1 / / VIS ExpMeter file

HIERARCH CAHA INS VIS ETALON SHUTTER $=11 / \mathrm{F}-\mathrm{P}$ shutter status in calunit

HIERARCH CAHA INS VIS ETALON UT-OPEN = / UTC at F-P shutter opening

HIERARCH CAHA INS VIS ETALON UT-CLOSE = / UTC at F-P shutter closing

HIERARCH CAHA INS VIS ETALON CURRENT $=/$ [A] F-P halogen lamp current

HIERARCH CAHA INS VIS ETALON VOLTAGE $=/$ [V] F-P halogen lamp voltage

HIERARCH CAHA INS VIS ETALON P-VALVE $=/$ [hPA] Pressure F-P valve (VIS-FP-S1)

HIERARCH CAHA INS VIS ETALON P-COMMON =/ [hPA] Pressure common NIR/VIS valve (NIR-VIS-FP-S2)

HIERARCH CAHA INS VIS ETALON T-THERMO $=/$ [OC] Internal temperature of thermopump

HIERARCH CAHA INS VIS CALUNIT OCTAGON = / OCtagon lamp mirror position

HIERARCH CAHA INS VIS CALUNIT LAMP = / Corresponding lamp

HIERARCH CAHA INS VIS CALUNIT LAMP-OK $=/$ Light coming from octagon

HIERARCH CAHA INS VIS CALUNIT FILTER SCIENCE = '' / 'A' wheel filter

HIERARCH CAHA INS VIS CALUNIT FILTER CALIBRA $=11 /$ 'B' wheel filter

HIERARCH CAHA INS VIS CALUNIT SOCKET NUMI $=/$ (UNO) Oth U-Ne lamp status

HIERARCH CAHA INS VIS CALUNIT SOCKET NUM2 $=/$ (UA1) 1st U-Ar lamp status

HIERARCH CAHA INS VIS CALUNIT SOCKET NUM3 $=/$ (UN1) 1st U-Ne lamp status

HIERARCH CAHA INS VIS CALUNIT SOCKET NUM4 $=/$ (TN1) 1st Th-Ne lamp status

HIERARCH CAHA INS VIS CALUNIT SOCKET NUM5 $=/$ (UA2) 2nd U-Ar lamp status

HIERARCH CAHA INS VIS CALUNIT SOCKET NUM6 $=/$ (UN2) 2nd U-Ne lamp status

HIERARCH CAHA INS VIS CALUNIT SOCKET NUM7 = / (TN2) 2nd Th-Ne lamp status

HIERARCH CAHA INS VIS CALUNIT SOCKET HALOGEN = 1'/ Flat-field hal lamp status

HIERARCH CAHA INS VIS CALUNIT SOCKET CURRENTI $=/$ [mA] Current of UNO

HIERARCH CAHA INS VIS CALUNIT SOCKET CURRENT2 $=/$ [mA] Current of UA1

HIERARCH CAHA INS VIS CALUNIT SOCKET CURRENT3 $=/$ [mA] Current of UN1

HIERARCH CAHA INS VIS CALUNIT SOCKET CURRENT4 $=/$ [mA] Current of TN1

HIERARCH CAHA INS VIS CALUNIT SOCKET CURRENT5 $=/$ [mA] Current of UA2

HIERARCH CAHA INS VIS CALUNIT SOCKET CURRENT6 $=/$ [mA] Current of UN2

HIERARCH CAHA INS VIS CALUNIT SOCKET CURRENT7 $=/$ [mA] Current of TN2

HIERARCH CAHA INS VIS CALUNIT SOCKET AGEI $=/[\mathrm{mA}$ h] Age of UNO

HIERARCH CAHA INS VIS CALUNIT SOCKET AGE2 $=/$ [mA h] Age of UA1

HIERARCH CAHA INS VIS CALUNIT SOCKET AGE3 $=/\left[\begin{array}{ll}\mathrm{mA} & \mathrm{h}\end{array}\right.$ Age of UN1

HIERARCH CAHA INS VIS CALUNIT SOCKET AGE4 $=/$ [mA h] Age of TN1

HIERARCH CAHA INS VIS CALUNIT SOCKET AGE5 $=/[\mathrm{mA} \mathrm{h}]$ Age of UA2

HIERARCH CAHA INS VIS CALUNIT SOCKET AGE6 $=/$ [mA h] Age of UN2

HIERARCH CAHA INS VIS CALUNIT SOCKET AGE7 $=/$ [mA h] Age of TN2

HIERARCH CAHA INS VIS CALUNIT SOCKET LAMPNUM1 $=/$ ID Of UNO

HIERARCH CAHA INS VIS CALUNIT SOCKET LAMPNUM2 $=/$ ID Of UAI

HIERARCH CAHA INS VIS CALUNIT SOCKET LAMPNUM3 $=/$ ID Of UN1

HIERARCH CAHA INS VIS CALUNIT SOCKET LAMPNUM4 $=/$ ID Of TN1

HIERARCH CAHA INS VIS CALUNIT SOCKET LAMPNUM5 $=/$ ID Of UA2

HIERARCH CAHA INS VIS CALUNIT SOCKET LAMPNUM6 $=/$ ID of UN2 


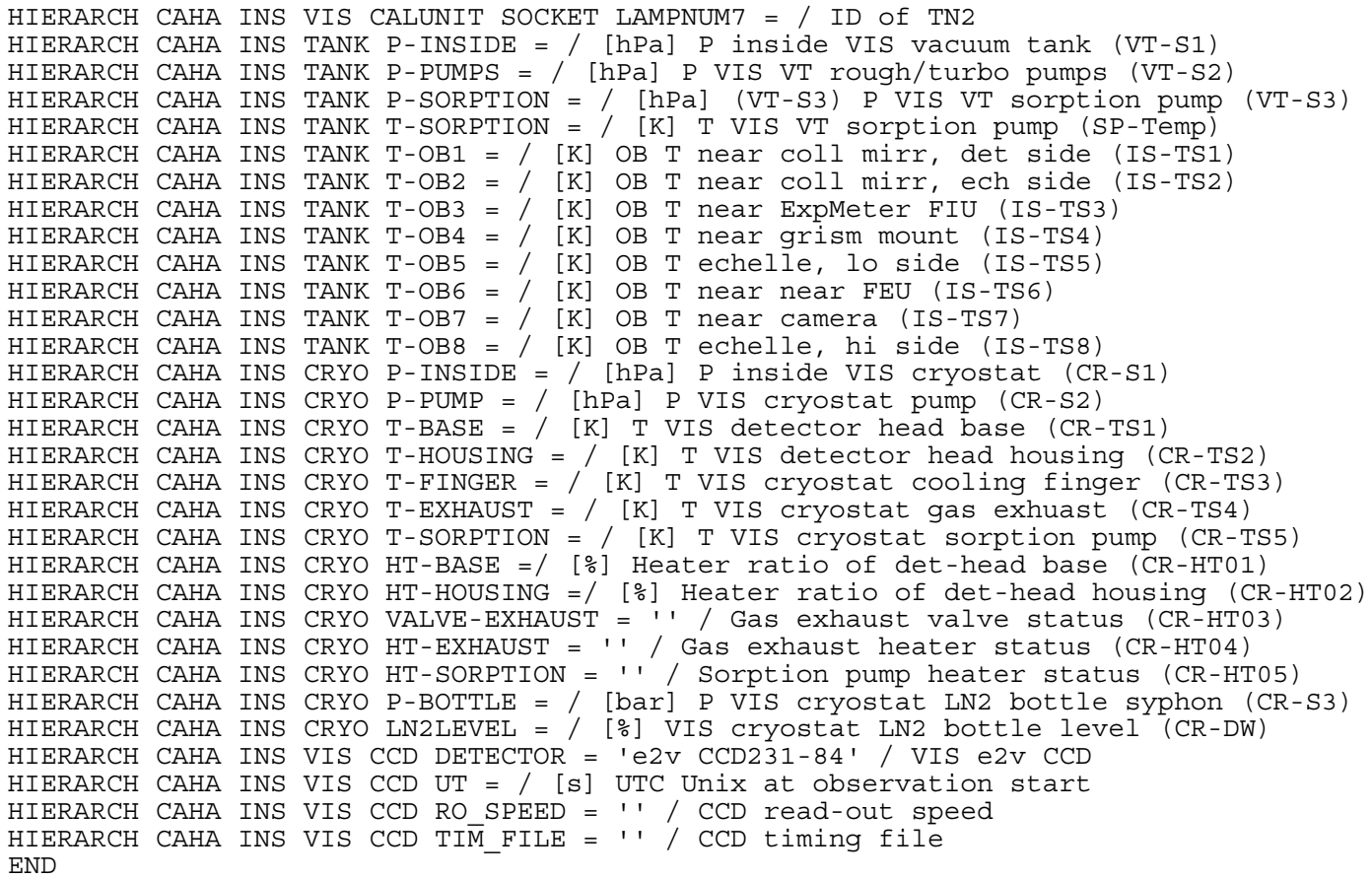

